\title{
La formación en derechos humanos y desarrollo sostenible de los docentes de educación primaria de la Ciudad de Buenos Aires
}

The training in human rights education and education for sustainable development for primary school teachers in the City of Buenos Aires

\author{
Alan Vogelfanger \\ Profesor Adjunto de Derecho Internacional de los Derechos Humanos \\ Facultad de Derecho \\ Universidad de Buenos Aires, Argentina \\ E-mail: avogelfanger@derecho.uba.ar
}

\begin{abstract}
Resumen: La adecuada preparación de maestros y profesores es fundamental para cumplir con el ODS4. Este artículo busca analizar la inserción curricular de contenidos de derechos humanos y desarrollo sostenible en los profesorados de formación docente de educación primaria de la Ciudad de Buenos Aires. Para eso, se indagó en los tres documentos clave que determinan los contenidos de la carrera: los Lineamientos Curriculares Nacionales para la Formación Docente Inicial, el Diseño Curricular Jurisdiccional elaborado por el Ministerio de Educación de la Ciudad de Buenos Aires, y el Plan Curricular Institucional desarrollado por los institutos públicos de formación
\end{abstract}


docente. Se destacaron los contenidos relevantes sobre derechos humanos o desarrollo sostenible y se establecieron indicadores temáticos para facilitar la sistematización y categorización de estos contenidos. Como resultado, si bien aparecen elementos relacionados con derechos humanos y desarrollo sostenible, sobre todo temas de diversidad, igualdad y no discriminación, no hay una perspectiva de derechos explícita. Además, la transversalidad de los contenidos permanece como un desafío. Asimismo, se concluye que la educación en derechos humanos y desarrollo sostenible necesitará trabajar en conjunto con otras disciplinas y otros ámbitos, por fuera de la educación y el derecho, para alcanzar sus nobles y ambiciosos objetivos.

Palabras clave: Educación en derechos humanos, educación en desarrollo sostenible, formación docente, educación primaria, ODS 4.

Abstract: A proper teacher training is crucial to achieve the SDG4. This article intends to analyse the curricular insertion of human rights and sustainable development contents in the career to become a primary teacher in the City of Buenos Aires. In order to do that, I investigated the three main documents that determine the contents for the carreer: the Lineamientos Curriculares Nacionales para la Formación Docente Inicial, the Diseño Curricular Jurisdiccional elaborated by the Ministry of Education of the City of Buenos Aires, and the Plan Curricular Institucional, written by the public institutes that offer the career to become a primary teacher. The relevant contents on human rights and sustainable development were highlighted and I used thematic indicators to facilitate the sistematization and categorization of those contents. The result was that even if there are elements about human rights and sustainable development in the documents, mainly related to diversity, equality and non-discrimination, there is no human rights perspective. Furthermore, the transversality of these contents remains as a challenge. It will also be concluded that human rights education and education for sustainable development will need to work and cooperate with other disciplines to achieve its noble and ambitious purposes.

Keywords: Human rights education, education for sustainable development, teacher training, primary education, SDG 4. 
Sumario: 1. Introducción. 2. La educación en derechos humanos y su vínculo con la educación en desarrollo sostenible. 3. La importancia de la formación docente. 4. Metodología del estudio. 5. El currículo del Profesorado de Educación Primaria de la Ciudad de Buenos Aires. 5.1. El Campo de la Formación General. 5.2. El Campo de la Formación Específica. 5.3. El Campo de la Formación en la Práctica Profesional. 5.4. Conclusiones sobre el currículo del Profesorado de Educación Primaria. 6. Palabras finales. 7. Referencias bibliográficas

\section{Introducción}

La educación en derechos humanos y en desarrollo sostenible es un derecho humano, reconocido en diversos tratados internacionales y en la meta 4.7 de la Agenda 2030. Además, es una herramienta fundamental para permitirles a las personas ejercer sus otros derechos humanos, para mejorar nuestra calidad de vida y para conseguir una sociedad más justa. Por eso, se entiende que esta clase de educación debe impartirse en todas las etapas de la educación formal, desde los niveles iniciales hasta la universidad y en los posgrados. Asimismo, la incorporación de estas temáticas y de estas perspectivas debe darse de manera transversal en toda la curricula de las instituciones educativas, y no limitarse a una materia o a un taller específico. Entonces, la formación en derechos humanos y en desarrollo sostenible corresponde a la totalidad de los docentes y a cualquier persona que esté involucrada en la enseñanza.

Por otra parte, la enseñanza de derechos humanos y desarrollo sostenible presenta desafíos particulares con respecto a la enseñanza de otros contenidos porque discute nuestros preconceptos, nos invita a reflexionar sobre las violaciones masivas y recurrentes de derechos humanos y porque involucra nuestra propia visión del mundo. A su vez, la enseñanza de estos temas muchas veces puede generar tensiones también hacia el exterior de las aulas, es decir, frente a autoridades escolares y familias. En consecuencia, la formación docente en estas temáticas es fundamental y los Estados, como garantes de los derechos humanos, deben cumplir un rol clave a la hora de establecer los parámetros, las normativas y las políticas educativas necesarias para llevar a cabo con éxito esta trascendental tarea. 
El presente artículo busca analizar la inserción curricular de contenidos de derechos humanos y desarrollo sostenible en los profesorados de formación docente de educación primaria de la Ciudad de Buenos Aires. La investigación es parte de un proyecto más amplio de Doctorado en Ciencias de la Educación, en la Facultad de Filosofía y Letras de la Universidad de Buenos Aires, que incluye no solo el análisis de la curricula de otras carreras de profesorado y educación superior, sino también una indagación más profunda, a través de la presencia de clases, entrevistas en profundidad y cuestionarios, que permitan conocer más allá de lo que presenta el plan de estudios. El presente estudio se basó en el análisis de documentos curriculares, sabiendo que la presencia de contenidos en derechos humanos o desarrollo sostenible en un programa de estudio no es condición suficiente para que exista un efectivo abordaje de la temática, aunque no deja de ser un factor relevante.

Para realizar el análisis se indagó en los Lineamientos Curriculares Nacionales para la Formación Docente Inicial, en el Diseño Curricular Jurisdiccional elaborado por el Ministerio de Educación de la Ciudad de Buenos Aires y en el Plan Curricular Institucional desarrollado por los institutos de formación docente. Así, se destacaron los contenidos relevantes sobre derechos humanos y/o desarrollo sostenible y se establecieron indicadores temáticos para facilitar la sistematización y categorización de estos contenidos, como se verá en la Tabla 4.

\section{La educación en derechos humanos y su vínculo con la educación en desarrollo sostenible}

La educación es un derecho humano por sí mismo, pero también es un elemento indispensable que permite realizar otros derechos humanos (Comité de Derechos Económicos Sociales y Culturales, 1999). La Relatora Especial de las Naciones Unidas sobre el derecho a la educación (2018) consideró que puede proteger a los niños de la violencia, la explotación, las actividades delictivas y las enfermedades, y que tiene el potencial de promover la paz y la tolerancia. En este sentido, "la educación cumple una función esencial, transformadora y de empoderamiento en la promoción de los valores de los derechos humanos" (Comité para la Eliminación de la Discriminación contra la Mujer, 2017, 1). El Tribunal Europeo de Derechos Humanos (2005; 2014; 2016) también sostuvo 
que el derecho a la educación es indispensable para el fomento y la promoción de los derechos humanos y que desempeña un papel fundamental en una sociedad democrática.

Adicionalmente, el derecho a la educación incluye necesariamente el derecho a recibir una educación en derechos humanos y también una educación en desarrollo sostenible. Algunos de los instrumentos internacionales que reconocen explícitamente el derecho a la educación y el derecho a la educación en derechos humanos y/o en desarrollo sostenible son: la Declaración Universal de Derechos Humanos (artículo 26), la Convención Internacional sobre la Eliminación de Todas las Formas de Discriminación Racial (artículo 7), el Pacto Internacional de Derechos Económicos, Sociales y Culturales (artículo 13), la Convención sobre la Eliminación de Todas las Formas de Discriminación contra la Mujer (artículo 10), la Convención sobre los Derechos del Niño (artículo 29), la Convención sobre los Derechos de las Personas con Discapacidad (artículos 8 y 24), la Convención Marco de las Naciones Unidas sobre el Cambio Climático (artículos 4 y 6) y el Acuerdo de París (artículo 12).

A su vez, a nivel regional de América se destacan, por ejemplo, el Protocolo Adicional a la Convención Americana sobre Derechos Humanos en materia de Derechos Económicos, Sociales y Culturales o "Protocolo de San Salvador" (artículo 13), la Convención Interamericana para prevenir, sancionar y erradicar la violencia contra la mujer o "Convención Belem do Pará” (artículo 6) y el Acuerdo Regional sobre el Acceso a la Información, la Participación Pública y el Acceso a la Justicia en Asuntos Ambientales en América Latina y el Caribe, más conocido como “Acuerdo de Escazú" (artículo 10).

La definición de educación en derechos humanos que se utilizará en este artículo es la indicada en la Declaración de las Naciones Unidas sobre educación y formación en materia de derechos humanos, de 2011, porque se trata de una de las definiciones más aceptadas en la actualidad (TIBBITTS, 2017). De este instrumento se pueden destacar algunos elementos centrales, como el objeto de promover el respeto de los derechos humanos, prevenir abusos e impulsar una cultura de derechos humanos. Además, la Declaración menciona el concepto de educación sobre derechos humanos (aspecto temático, de contenidos), por medio de o a través de los derechos humanos (enseñar con 
el ejemplo, coherencia entre lo que se enuncia y cómo se actúa), y para los derechos humanos (propósito transformador, de incidencia, influencia).

Ahora bien, tribunales internacionales como la Corte Interamericana de Derechos Humanos han sostenido recientemente que "existe un amplio reconocimiento sobre la relación interdependiente entre la protección al medio ambiente, el desarrollo sostenible y los derechos humanos" (Corte IDH, 2017, párr.52; Corte IDH, 2020, párr.85). La Comisión Interamericana de Derechos Humanos (2019) también sostuvo específicamente que "la relación estrecha entre los derechos humanos, el desarrollo sostenible y el medio ambiente abarca innumerables facetas y alcances" (CIDH, 2019, 35). Es decir, en la actualidad, no es posible imaginar la protección de los derechos humanos sin incluir el ambiente.

La iniciativa mundial más actual y de mayor envergadura que probablemente mejor refleja la interacción entre los derechos humanos y el ambiente es la Agenda 2030. Este programa, adoptado en 2015 desde la ONU, incluye 17 Objetivos de Desarrollo Sostenible (ODS) que abarcan numerosas metas y una guía común para todos los países. Los propósitos generales incluyen erradicar la pobreza, proteger el planeta y asegurar la prosperidad para todas las personas. Como se adelantó previamente, si bien no es una fuente jurídica vinculante, la Agenda 2030, en su meta 4.7, indica explícitamente que los Estados deben garantizar la educación para el desarrollo sostenible y los derechos humanos. Uno de los indicadores para monitorear su cumplimiento es el grado en que la educación para la ciudadanía global y la educación para un desarrollo sostenible (incluyendo la igualdad de género y la educación en derechos humanos) se incorporan en las políticas nacionales de educación, los planes de estudio, la formación de docentes y las evaluaciones de estudiantes. En particular, con respecto a la formación docente, el ODS 4 también incluye como una de sus metas el aumento considerable de la oferta de docentes calificados. A su vez, el ODS 12 (en particular, la meta 12.8) impulsa a los Estados a asegurar que las personas tengan la información y los conocimientos pertinentes para el desarrollo sostenible. El ODS 13 (meta 13.3) también pretende mejorar la educación y la sensibilización, en particular, sobre la mitigación y adaptación al cambio climático. 
Básicamente, la educación en derecho sostenible a la que se hace referencia en este artículo implica incluir en la enseñanza aspectos de cambio climático, biodiversidad, consumo responsable y sustentable, entre otros contenidos. Como define UNESCO (2016a, 25), además, "la educación en desarrollo sostenible empodera a los educandos para tomar decisiones informadas y adoptar acciones responsables en favor de la integridad del ambiente, la viabilidad de la economía y una sociedad más justa, para las generaciones actuales y futuras, respetando la diversidad cultural". El objetivo primordial es facilitar una transición hacía economías y sociedades más verdes, con educandos equipados con habilidades para trabajar en rubros ambientalmente amigables y motivados para adoptar modelos de vida más sustentables. Entonces, la educación en desarrollo sostenible comprende la educación ambiental, así como elementos de economía, pobreza, población, salud y seguridad alimenticia, entre otros.

La educación en derechos humanos y la educación en derecho sostenible comparten numerosos objetivos, entre ellos, impulsar cambios individuales y colectivos, modificar valores, actitudes y comportamientos, y promover el pensamiento crítico y la participación ciudadana, siempre con el objetivo final de mejorar la calidad de vida de las personas y de alcanzar una sociedad más justa. Por lo tanto, garantizar la educación, y asegurar que esa educación contenga elementos de derechos humanos y desarrollo sostenible, no solo es una obligación de los Estados que se desprende del Derecho Internacional de los Derechos Humanos sino que es trascendental por los beneficios que genera a nivel individual y colectivo en las personas y la sociedad.

\section{La importancia de la formación docente}

Uno de los principales puntos sobre los que existe un gran consenso es que la educación en derechos humanos y en desarrollo sostenible debe atravesar todos los niveles de la educación formal y, para que sea efectiva, no alcanza con que exista una sola materia aislada que abarque estos temas; es clave que el contenido y el enfoque de los derechos humanos aparezcan de manera transversal (IIDH, 2013; RONCONI, 2017; MEDICI, 2018). Lo mismo aplica para la educación en desarrollo sostenible, que debe integrarse al currículum escolar en su conjunto, en todas las disciplinas (BACHMANN, 2008; CARMONA LARA, 2010; BARAJAS, 2012; CONDENANZA y CORDERO, 2013). El 
objetivo final es considerar a los derechos humanos y al desarrollo sostenible como un prisma a través del cual miramos la realidad.

Entonces, una de las primeras consecuencias de esto es que la educación en derechos humanos y en desarrollo sostenible corresponde a todos los educadores, no solo a quienes enseñan cursos sobre estas temáticas en particular; es decir, implica a toda persona que esté involucrada en los procesos de enseñanza y aprendizaje (RONCONI, 2017). En otras palabras, todo el personal docente tiene una responsabilidad esencial de transmisión de valores y debe proporcionar una educación comprometida con la construcción de la paz, la defensa de los derechos humanos y la democracia, la erradicación de la pobreza y la discriminación, el desarrollo sostenible y el entendimiento multicultural (ACNUDH y UNESCO, 2012).

Esto, además, es particularmente cierto para la educación primaria. El objetivo de estas instituciones no es solamente brindar conocimientos teóricos sino formar personas, con valores y herramientas para vivir adecuadamente en una sociedad democrática y transformar el mundo en un lugar mejor. De hecho, para un porcentaje elevado de docentes, formar ciudadanos críticos, responsables y pensantes es la meta principal de la escuela (ALLIAUD y VEZUB, 2012). Más aún, hay autores que sostienen que "hay una total unanimidad entre los educadores acerca de la importancia radical de conocer la existencia de los derechos humanos, precisamente, en los niveles básicos y obligatorios de la enseñanza" (GIL CANTERO, 1991, 537).

Sin embargo, la formación docente en estos temas aún permanece como un desafío. KLAINER y FERNÁNDEZ (2008), por ejemplo, advirtieron que en Argentina la ausencia de una capacitación sistemática a docentes y de materiales didácticos adecuados representan una de las mayores dificultades para implementar esta clase de educación. En el campo ambiental, GONZÁLEZ GAUDIANO (2005) también diagnosticó que faltaba atención en la formación de docentes. Por otra parte, la educación en derechos humanos y en desarrollo sostenible puede significar un reto mayor que la enseñanza de la mayoría de las otras materias académicas (Instituto Danés de Derechos Humanos, 2013). Esto es así porque plantea preguntas complejas acerca del comportamiento humano y la diversidad cultural, porque busca dar respuestas a por qué han sido denegados los derechos de las personas, porque se comprometen nuestros valores, sentimientos y 
opiniones, y porque se desafían seriamente las visiones y prejuicios acerca del mundo (Instituto Danés de Derechos Humanos, 2013). Como sintetiza BACHMANN, "si la realidad es compleja en sí misma, su enseñanza también lo es” $(2008,10)$. Precisamente por eso es tan importante la formación docente en estos temas.

En este sentido, trabajos que incluyeron entrevistas y encuestas a docentes de Argentina reflejaron que muchos no se sienten preparados o capacitados para trabajar, enseñar e interactuar con la subjetividad de los alumnos en las aulas (VEZUB, 2011; VEZUB y GARABITO, 2017), un aspecto que es fundamental para la educación en derechos humanos y desarrollo sostenible ya que "se trata de saberes que deben interpelar subjetivamente a los estudiantes" (MASTACHE, 2019, 174). UNESCO (2016b, 13) también consideró, en particular, que tanto los educadores encargados de la atención y educación de la primera infancia como los docentes de primaria y secundaria no cuentan con las competencias y capacidades para incorporar, integrar y poner en práctica la educación en desarrollo sostenible, por lo que se requiere "una mayor innovación en la formación del personal y en los establecimientos de enseñanza para transformar los planes de estudios y la pedagogía de los institutos de enseñanza superior”. A su vez, como señalan MAGENDZO y TOLEDO-JOFRÉ “el profesorado debe estar capacitado para enfrentar posibles dificultades y resistencias que se presentan en las instituciones educacionales, su personal directivo y padres y madres de familia cuando se enseñan 'temas controversiales"” $(2015,13)$. Es decir, es particularmente relevante que quienes deban impartir esta clase de conocimientos en las escuelas estén adecuadamente preparados para enfrentar desafíos tanto internos del aula como externos.

Más aún, las formas en que se enseñen derechos humanos y desarrollo sostenible pueden influenciar y ser determinantes en la postura que el estudiantado adopte frente estos temas (REQUESENS GALNARES, 2006; Instituto Danés de Derechos Humanos, 2013). En consecuencia, es fundamental que los docentes cuenten con una buena formación al respecto y que tengan buenas herramientas pedagógicas para abordar estos temas porque de ellos depende, en gran parte, la actitud que los estudiantes tomen ante estas cuestiones tan relevantes.

\section{Metodología del estudio}


El presente artículo pretende estudiar la inserción curricular actual de contenidos de derechos humanos y desarrollo sostenible en los profesorados de formación docente de educación primaria de la Ciudad de Buenos Aires, una instancia de educación superior. En esta jurisdicción, cada carrera de profesorado cuenta con un Diseño Curricular Jurisdiccional (DCJ), elaborado por el Gobierno de la Ciudad, para que sirva como guía para las instituciones que ofrezcan esos profesorados. A su vez, cada instituto cuenta con un Plan Curricular Institucional (PCI), a través de cual realiza la adaptación propia al DCJ.

Este artículo se enfocará exclusivamente en los institutos de formación docente públicos. Esto no quiere decir que el Estado, como garante de los derechos humanos, no deba tener un rol de control y supervisión sobre la educación privada. El Gobierno de la Ciudad de Buenos Aires también debe asegurar que las instituciones privadas que forman docentes brinden una educación en derechos humanos y desarrollo sostenible para que luego sea transmitida a sus futuros alumnos. No obstante, el análisis de esta obligación de monitoreo sobre las instituciones privadas quedó por fuera del estudio de esta investigación.

Para este caso, existen 11 institutos públicos que ofrecen la Carrera de Profesorado de Educación Primaria, y todos conciliaron un único Plan Curricular Institucional. Es decir, todas las instituciones públicas que ofrecen la carrera de profesorado en educación primaria tienen el mismo PCI. Entonces, el presente artículo se concentrará en el DCJ y en el PCI aplicable a los institutos públicos que ofrecen el profesorado de educación primaria.

Los DCJ cuentan con una introducción, donde se desarrollan los objetivos, la fundamentación pedagógica, el perfil de los egresados, la organización de la carrera y los lineamientos generales del profesorado. Posteriormente, se presenta una división entre el Campo de la Formación General (CFG), el Campo de la Formación Específica (CFE) y el Campo de la Formación en la Práctica Profesional (CFPP).

El CFG tiene como propósito establecer un marco conceptual común, que permita analizar y comprender el contexto del trabajo docente desde diversas perspectivas (histórica, filosófica, artístico-expresiva, política, social, pedagógica, didáctica y 
psicológica); el CFE aporta herramientas para orientar, diseñar y poner en marcha el plan de enseñanza de disciplinas específicas, la selección de los contenidos, los tipos de evaluación, y el uso del tiempo y de los espacios, entre otros; finalmente, el CFPP busca que los futuros docentes adquieran a través de la práctica las capacidades necesarias para el desempeño en las instituciones educativas, integrando y actualizando los conocimientos y habilidades adquiridos en los otros dos campos (PCI de Educación Primaria, 2015). Además, están en los DCJ los Espacios de Definición Institucional (EDI), de forma tal de que cada profesorado tenga cierto margen para incluir aspectos, contenidos o herramientas propias de su institución.

El estudio del DCJ y del PCI requirió una lectura y un análisis meticuloso ya que los contenidos buscados pueden no aparecer explícitamente con los nombres de "derechos humanos" o "desarrollo sostenible". Por ejemplo, las referencias a derechos humanos pueden aparecer como derechos fundamentales, libertades fundamentales o derechos elementales. Lo importante, entonces, fue identificar qué temas existen en la curricula que pueden ser comprendidos dentro de las definiciones de educación en derechos humanos y educación en desarrollo sostenible, independientemente de su denominación en el documento.

Adicionalmente, se adoptaron indicadores temáticos. Esto permitió, además de cuantificar y clasificar los contenidos presentes, observar cuáles son las áreas más repetidas en el plan de estudio y cuáles necesitarían mayor desarrollo. Para seleccionar los indicadores se tomó como base el III Informe Interamericano de la Educación en Derechos Humanos, elaborado por el Instituto Interamericano de Derechos Humanos (2004), y se lo actualizó/adaptó de acuerdo a las necesidades presentadas por el DCJ y el PCI.

Para la presente investigación, en síntesis, se eligieron los siguientes indicadores: 1 . Normativa sobre educación (contenidos relacionados con la Ley Nacional de Educación, la Constitución Nacional y de la Ciudad, los tratados internacionales y otras leyes que regulen la educación); 2. Democracia, ciudadanía, participación social y orden institucional nacional e internacional (elementos de derechos políticos, organización del Estado, formas de gobierno, organizaciones internacionales como Naciones Unidas o la OEA); 3. Género, diversidad, inclusión y multiculturalismo (derechos de las mujeres, 
minorías sexuales, aspectos de religión, raza, derechos de los pueblos originarios, tolerancia y bullying, entre otros); 4. Derechos de Niños, Niñas y Adolescentes (sobre todo los enunciados en la Convención de los Derechos del Niño.); 5. Ambiente y ecologismo (aspectos de biodiversidad, cambio climático, desarrollo sostenible, contaminación y protección de los recursos naturales); 6. Memoria, Verdad y Justicia (justicia transicional, dictadura, terrorismo de Estado); 7. Salud, Educación Sexual Integral (ESI) y Adicciones (salud sexual y reproductiva, prevención y respuesta ante adicciones y consumos problemáticos de drogas y alcohol); 8. Derechos Económicos, Sociales, Culturales y Derechos Colectivos (derechos laborales, sindicales, derechos de consumidores); 9. Derechos de las Personas con Discapacidad; y 10. Pedagogía de los derechos humanos (formas de enseñanza sobre estos temas, recursos pedagógicos).

Además de relatar los aspectos más importantes del DCJ y del PCI de cada una de las materias de la carrera, a medida que se detectaban contenidos de derechos humanos o de desarrollo sostenible, la información era volcada en la Tabla 4, señalando dentro de qué indicadores temáticos estaban incluidos y el número de página del documento donde aparecía.

\section{El currículo del Profesorado de Educación Primaria de la Ciudad de Buenos Aires}

Antes de ingresar en el estudio de los documentos correspondientes de la Ciudad de Buenos Aires, es necesario mencionar que para el Profesorado de Educación Primaria existe un documento a nivel nacional, aplicable a todas las provincias de Argentina, que sirve como marco regulatorio de los diseños curriculares jurisdiccionales de todo el país: los Lineamientos Curriculares Nacionales para la Formación Docente Inicial (no existen lineamientos similares para los profesorados de educación secundaria). Allí, ya se habla de la necesidad de "preparar docentes con capacidad de enseñar, generar y transmitir los valores necesarios de las personas para el desarrollo nacional y la construcción de una sociedad más justa" (Resolución CFE N²4/07, 2007, 1). También aparecen contenidos de igualdad y se afirma que se concibe a la educación como un bien público y un derecho personal y social, y como una prioridad nacional para profundizar el ejercicio de la ciudadanía democrática y el respeto de los derechos humanos (Resolución CFE N 24/07, 2007). 
Los Lineamientos también pretenden garantizar los derechos docentes y promover valores como la libertad, el respeto a la diversidad, la solidaridad, la justicia y el bien común. Además, el documento aspira a promover la transversalidad de estos valores, la interdisciplinariedad y el diálogo. Finalmente, también aparecen incentivos a la reflexión y al pensamiento crítico y a considerar los elementos culturales de los pueblos originarios. No obstante, dentro de las recomendaciones temáticas que se realizan, no se sugiere la incorporación de espacios específicos sobre derechos humanos ni desarrollo sostenible.

Ahora bien, con respecto a la introducción del Diseño Curricular Jurisdiccional para el Profesorado en Educación Primaria de la Ciudad de Buenos Aires, se destacan algunos elementos que también podrían estar incluidos en el concepto de educación en derechos humanos. Por ejemplo, "se considera de especial importancia la existencia de la inclusión social en todos sus aspectos y se sostiene que la escuela debe ser una escuela abierta a la igualdad de oportunidades" (DCJ Educación Primaria, 2014, 3). Luego, explica que es necesario "destacar el paradigma de la inclusión y la diversidad de tal forma que atraviese los contenidos y las prácticas a fin de consolidar un perfil profesional docente que pueda llevar adelante una enseñanza efectiva y equitativa para todos los alumnos" (DCJ Educación Primaria, 2014, 4).

Sin embargo, en el DCJ no hay materias, asignaturas o unidades curriculares específicas sobre derechos humanos ni desarrollo sostenible. Esta falencia será corregida por los propios institutos de formación docente a través del Plan Curricular Institucional, donde se incluye la materia Ética, derechos humanos y construcción de la ciudadanía. Sí se destaca tanto en el DCJ como en el PCI la unidad curricular sobre Educación Sexual Integral (ESI), que está explícitamente vinculada a la educación en derechos humanos.

\subsection{El Campo de la Formación General}

Tabla 1: Campo de la Formación General (DCJ Educación Primaria, 2014, 17).

\section{UNIDADES CURRICULARES}

\begin{tabular}{|c|c|}
\hline Pedagogía & Trabajo/Profesionalización Docente \\
\hline Psicología Educacional & Nuevas Tecnologías \\
\hline Historia Social y Política de la Educación & Nuevos Escenarios: Cultura, Tecnología y \\
Argentina & Subjetividad \\
\hline Instituciones Educativas & Educación Sexual Integral (ESI) \\
\hline Filosofía y Educación & Trabajo de Campo \\
\hline Didáctica I y Didáctica II & Espacios de Definición Institucional \\
\hline
\end{tabular}


En el DCJ, dentro de la unidad curricular de Pedagogía, se vuelve a destacar la importancia de la diversidad. Por ejemplo, se afirma que "a pesar de que actualmente se puede caracterizar el escenario escolar -y también extraescolar-como injusto y desigual, creemos que la escuela sigue siendo uno de los pocos lugares en donde se pueden producir prácticas escolares basadas en el respeto a las diferencias (de clase, de género, de etnia, de religión o de orientación sexual), en la justicia, en la solidaridad, en la empatía con el otro/a y en la igualdad" (DCJ Educación Primaria, 2014, 23). En Psicología Educacional, se incluye la problemática de las adicciones, drogas y alcohol, y también se pretende trabajar con la "caracterización de los colectivos de personas que encuentran barreras para el aprendizaje (personas con discapacidad intelectual, emocional, física, sensorial, con desventajas socioculturales)" (DCJ Educación Primaria, 2014, 26). En la unidad curricular Historia Social y Política de la Educación Argentina si bien se habla de promover el análisis crítico y tener un enfoque social, no hay menciones a los derechos humanos, a su historicidad, a su lucha y sus conquistas, ni al desarrollo sostenible ni a la importancia de los aspectos ambientales en nuestro mundo.

En cuanto a estas primeras tres unidades, el PCI en Pedagogía vuelve a hacer referencia al tema de la diversidad y las desigualdades socioeconómicas. En Psicología Educacional prácticamente no hay referencias a cuestiones que puedan involucrar derechos humanos ni desarrollo sostenible; de hecho, no aparecen las referencias a la temática de adicciones, drogas y alcohol que sí están presentes en el DCJ. En Historia Social y Política de la Educación Argentina si bien se explica brevemente el desarrollo de la educación en Argentina, con menciones al terrorismo de estado y a cuestiones laborales y sindicales, se trata más bien de un relato histórico, sin explicitar conexiones con los derechos humanos.

En Instituciones Educativas, el DCJ vuelve a incluir temas de adicciones, drogas, campañas de promoción y prevención, y agrega también aspectos de pobreza y de diversidad/inclusión (DCJ Educación Primaria, 2014). En Filosofía y Educación no hay nada relacionado con derechos humanos o desarrollo sostenible, y en Didáctica I y Didáctica II, si bien aparecen técnicas de enseñanza para considerar la diversidad de los alumnos, no hay ninguna particularidad sobre derechos humanos ni desarrollo sostenible a pesar de que, como se señaló anteriormente, la enseñanza de estas cuestiones requiere destrezas particulares. El PCI, por su parte, mantiene contenidos de violencia y de pobreza 
en Instituciones Educativas, pero vuelve a ocultar la problemática de adicciones. En Filosofía y Educación tampoco hay elementos de derechos humanos o desarrollo sostenible y en Didáctica I y II, aunque se hable sobre métodos de enseñanza, no aparece nada específico sobre derechos humanos.

La unidad curricular Trabajo/Profesionalización Docente, en el DCJ, incluye contenidos sobre los derechos y deberes de los docentes y sobre la normativa existente. En Nuevas Tecnologías aparece cierta perspectiva de derechos al considerar la convivencia en el ciberespacio, el uso seguro, responsable y solidario de internet, el respeto a la diversidad, la protección de la información y de los datos personales, y la accesibilidad para alumnos con discapacidades (DCJ Educación Primaria, 2014, 38). En Nuevos Escenarios: Cultura, Tecnologías y Subjetividad solamente aparece alguna mención sobre la interculturalidad, la transculturalidad y la multiculturalidad. Trabajo de Campo no presenta un foco de derechos humanos o desarrollo sostenible.

En el PCI, también aparece un desarrollo sobre los derechos laborales y sindicales en Trabajo Docente. En particular, se destaca la salud laboral y se propone estudiar el tema explícitamente "en el marco del respeto a los derechos humanos" (PCI de Educación Primaria, 2015, 39). También se aborda "la educación como construcción de ciudadanía" (PCI de Educación Primaria, 2015, 38). En la unidad Nuevas Tecnologías se receptan los elementos sobre la seguridad en internet, la prevención de riesgos, ciberbullying, privacidad y protección de datos personales señalados en el DCJ. En Nuevos Escenarios: Cultura, Tecnologías y Subjetividad sí aparecen conexiones con, por ejemplo, "la exclusión a gran escala, la administración de justicia, el gobierno de lo público, el funcionamiento de las instituciones, la participación democrática, y los modos de atención de las demandas sociales" (PCI de Educación Primaria, 2015, 34). También dentro de los propósitos de esta asignatura en el PCI se busca "favorecer el análisis de transformaciones sociales y tendencias profundas de cambio" (PCI de Educación Primaria, 2015, 36). La unidad Trabajo de Campo tampoco tiene en el PCI ningún contenido con derechos humanos ni desarrollo sostenible. Por último, está la unidad curricular Lenguajes artísticos expresivos, que llena el Espacio de Definición Institucional. Aquí se busca promover, entre otros, "el reconocimiento de las diferentes agendas culturales disponibles: museos, exposiciones, conciertos" (PCI de Educación Primaria, 2015, 51). 
Se dejó para el final el análisis de la unidad curricular Educación Sexual Integral, por ser la más vinculada con la educación en derechos humanos. Explica el DCJ: "la presencia de la Educación Sexual Integral (ESI) en las escuelas reafirma la responsabilidad del Estado y la escuela en la protección de los derechos de los niños, niñas y adolescentes como también su capacidad de generar condiciones para igualar el acceso a la información y a la formación" (DCJ Educación Primaria, 2014, 41). En cuanto al contenido, se indica que "la educación sexual debe: enseñar a conocer, valorar, respetar y cuidar de uno mismo y de los demás; relacionarse con los otros de manera solidaria y en el marco del respeto por las diferencias; reconocer y expresar pensamientos, sentimientos y afectos; comunicarse con el otro; enfrentar y resolver los problemas y los conflictos que se plantean en la vida cotidiana; poner límites para protegerse ante situaciones de maltrato y abuso; desarrollar la autoestima en la construcción de la identidad y la autonomía en la toma de decisiones; orientar el trabajo reflexivo sobre género" (DCJ Educación Primaria, 2014, 42). Desde ya, muchos de estos valores deben enseñarse siempre y ser transversales, sin estar limitados solo a la ESI.

El DCJ luego muestra un apartado específico sobre derechos humanos dentro de esta unidad curricular. Aquí se explica que "enmarcar la ESI en los derechos humanos es reconocer la importancia que estos tienen en la formación de sujetos de derecho, la construcción de la ciudadanía y la reafirmación de los valores de la democracia” (DCJ Educación Primaria, 2014, 43). Más aún, habla de "la responsabilidad del Estado por garantizar el acceso a contenidos curriculares y revalorizar el rol de los docentes en el cumplimiento de dichos derechos" (DCJ Educación Primaria, 2014, 43). También se llama a tener en cuenta la situación de alumnos con discapacidad, la enseñanza de derechos sexuales y reproductivos y de conceptos como identidad, sexo, género, identidad sexual, orientación sexual, y a trabajar sobre la deconstrucción de prejuicios y estereotipos. Además, se encuentra en el DCJ la enseñanza de los "recursos existentes en la CABA ante situaciones de vulneración de derechos" (DCJ Educación Primaria, 2014, 45), que es un aspecto fundamental de la educación sobre derechos humanos, que permite llegar a la educación para los derechos humanos. Por último, el documento llama a utilizar un "enfoque de derecho en la práctica cotidiana de la escuela" (DCJ Educación Primaria, $2014,45)$. Esto desde ya es valioso, pero no se ve en el marco de las otras materias. Es decir, por más que esta pretensión de transversalidad aparezca dentro de la unidad 
curricular sobre ESI, si no se recepta explícitamente en las otras unidades curriculares, difícilmente se aplique el enfoque de derechos.

En cuanto al PCI, se repite el mismo fraseo en cuanto la responsabilidad del Estado en la protección de derechos de niños, niñas y adolescentes, y sobre lo que debe enseñar la educación sexual. También se encuentra presente el apartado específico sobre derechos humanos, con referencias explícitas a las normas internacionales y locales, a evitar estereotipos y discriminación, y a brindar información y herramientas para intervenir en situaciones de vulneración de derechos.

\subsection{El Campo de la Formación Específica}

Tabla 2: Campo de la Formación Específica (DCJ Educación Primaria, 2014, 18).

BLOQUES CURRICULARES

Enseñanza de la Matemática en la Educación Primaria

Enseñanza de la Lengua y la Literatura en la Educación Primaria

Enseñanza de las Ciencias Naturales en la Educación Primaria

Enseñanza de las Ciencias Sociales en la Educación Primaria

\section{UNIDADES CURRICULARES}

Tecnologías de la Información y la Comunicación en la Educación Primaria

Sujetos de la Educación Primaria - La

Psicología y Cultura del Niño en la Educación Primaria

Problemáticas de la Educación Primaria

Espacios de Definición Institucional

El bloque sobre Enseñanza de la Matemática en la Educación Primaria no contiene ninguna mención que pueda relacionarse con temas de derechos humanos o desarrollo sostenible, ni en el DCJ ni en el PCI. Aunque parezca difícil incorporar esta clase de conocimientos en esta materia, al menos desde los ejemplos utilizados o la formulación de problemas, es posible y necesario incorporar un enfoque de derechos, en cualquier asignatura.

En el bloque Enseñanza de la Lengua y la Literatura en la Educación Primaria sí aparecen algunos contenidos que podrían vincularse con derechos humanos. Por ejemplo, se explica que "se lee para entretenerse, para identificarse con los sentimientos de otros u oponerse a ellos, para entender cómo se vive en lugares desconocidos, para conocer las ventajas de consumir determinados alimentos o para analizar el discurso de otros cuyos puntos de vista se requiere comprender; se escribe para pedir disculpas por un error involuntario, comunicarse con otros a distancia, para plantear un punto de vista diferente ante un conflicto, emitir una opinión fundamentada" (DCJ Educación Primaria, 2014, 
50). Además, vuelven a aparecer cuestiones de participación, diversidad y discriminación, y se pretende fomentar el espíritu crítico a través de la "iniciación de la lectura crítica de la prensa" (DCJ Educación Primaria, 2014, 51). En el PCI, se receptan en general estos elementos.

En Enseñanza de las Ciencias Naturales es quizás donde aparece la mejor oportunidad para incluir la educación en desarrollo sostenible. Precisamente, el DCJ anuncia que "de lo que se trata es de imprimir en la enseñanza de las ciencias naturales un contexto humanista que abarque los aspectos sociales, culturales, políticos y económicos en su totalidad" (DCJ Educación Primaria, 2014, 54). Asimismo, continúa con la intención de incorporar "la discusión de valores tales como la equidad, la justicia social y la sustentabilidad ambiental" (DCJ Educación Primaria, 2014, 55), y temas como "la vida y su biodiversidad, la promoción y el cuidado de la salud" (DCJ Educación Primaria, 2014, 57). En el PCI se reiteran los puntos sobre la enseñanza en el contexto humanista, sobre equidad, justicia social, sustentabilidad y biodiversidad, y se agrega también el análisis de los impactos ambientales para "tomar conciencia y diferenciar cuáles se deben a fenómenos y sucesos naturales, y cuáles son consecuencia de actividades económicas promovidas por intereses sectoriales” (PCI de Educación Primaria, 2015, 82).

Así como el bloque anterior representaba la oportunidad más clara para incluir la educación en desarrollo sostenible, el bloque Enseñanza de las Ciencias Sociales sería la mejor ocasión para incorporar explícitamente la educación en derechos humanos. Efectivamente, el DCJ establece propósitos sumamente ligados con los objetivos de la educación en derechos humanos. El documento, por ejemplo, señala la necesidad de que los futuros docentes asuman un compromiso como miembros de la sociedad y tomen conciencia de la importancia del conocimiento social para comunicarse con los demás, escuchar, desarrollar argumentos, para establecer consensos y "para colaborar en el fortalecimiento de una democracia y una ciudadanía participativa, con vistas a la construcción de realidades socialmente cada vez más justas y solidarias, económica y culturalmente más distributivas de los bienes y del conocimiento, respetuosas de la diversidad” (DCJ Educación Primaria, 2014, 58).

También el DCJ propone que los estudiantes revisen "sus propias representaciones, prejuicios y preconceptos sobre el mundo social y su enseñanza" (DCJ Educación 
Primaria, 2014, 59). Más aún, dentro de los ejes de contenidos propuestos están "los recursos naturales, formas de apropiación y explotación y problemáticas asociadas” y “distintas formas de organización de las sociedades indígenas en el territorio americano: relaciones entre aspectos políticos, sociales, económicos y creencias” (DCJ Educación Primaria, 2014, 59). Otros ejes temáticos del bloque son "las transformaciones sociales, políticas, económicas y culturales relacionadas con los procesos migratorios", "riesgos, vulnerabilidad y desastres sociales" y "origen, transformaciones y crisis del capitalismo" (DCJ Educación Primaria, 2014, 60). La propuesta de temas está muy conectada con la educación en derechos humanos; no obstante, no hay un enfoque de derechos ni una conexión explícita con los derechos humanos o con el desarrollo sostenible.

En el PCI también se recepta la necesidad de que los futuros docentes sean protagonistas de la sociedad y la ciudadanía. Además, están presentes los elementos de diversidad y respeto por las otras personas, se enfatiza la enseñanza para la democracia y la participación, y se reiteran los ejes sobre recursos naturales, pueblos originarios, procesos migratorios, vulnerabilidad y desastres sociales, integración y exclusión, y crisis del capitalismo. Por último, se destacan numerosos elementos en relación con los recursos pedagógicos necesarios para transmitir y construir esta clase de conocimientos. Para comenzar, se propone una "reflexión acerca del para qué, el qué y el cómo de la enseñanza de determinados contenidos" (PCI de Educación Primaria, 2015, 98). A su vez, se propone específicamente el uso de debates, simulaciones y juegos de rol, que promueven la tolerancia, el respeto y la escucha hacia la otra persona, y visitas a museos, instituciones públicas y privadas u otros organismos sociales, políticos y culturales, que son cruciales para bajar el contenido a la realidad y conectar los saberes con aspectos propios del lugar donde viven.

El PCI, en el bloque Enseñanza de las Ciencias Sociales, incluye además la asignatura Ética, Derechos Humanos y Construcción de Ciudadanía (que no está presente en el DCJ). Si bien se aclaró que la existencia de una asignatura específica no es suficiente para alcanzar una apropiada educación en derechos humanos y desarrollo sostenible, en muchos casos es un primer paso necesario para lograr la inserción curricular transversal de estos contenidos. 
Desde el comienzo de la descripción de la materia se anuncia que el objetivo es "formar a los futuros docentes para la enseñanza de los conocimientos y las competencias básicas para convivir en una sociedad democrática y plural, en la que el ejercicio de la ciudadanía incluya como marco de referencia, además de los aspectos civiles, políticos y sociales, los principios universales expresados en los diferentes instrumentos internacionales y nacionales sobre los derechos humanos" (PCI de Educación Primaria, 2015, 104). Acá nuevamente se propone trabajar en forma transversal con otras áreas. Incluso se dice explícitamente que "los derechos humanos constituyen una perspectiva ética que debería estar presente en la enseñanza de cualquier asignatura, dado que constituyen un marco para las relaciones en el aula y el ejercicio de la autoridad docente" (PCI de Educación Primaria, 2015, 107). Sin embargo, como se dijo anteriormente, si esta intención solo se expresa en el bloque de Ciencias Sociales y en la unidad curricular de Educación Sexual Integral, pero no se reitera en los otros bloques o unidades curriculares, se corre el riesgo de que, justamente, solo quede en una buena intención.

El desarrollo de la unidad contiene elementos de construcción de ciudadanía, educación ambiental, cuidado de los recursos naturales, consumo responsable, relación entre un ambiente sano y la garantía de derechos económicos y sociales, derechos de niños, niñas y adolescentes, derechos de consumidores, género, diversidad, igualdad y no discriminación, derechos de las mujeres, migrantes, pobreza, pensamiento crítico, democracia, tolerancia, solidaridad, normativa internacional y nacional, participación, Estado de Derecho, terrorismo de Estado, memoria, verdad y justicia, y mecanismos de protección de derechos, entre otros. Es decir, el componente de educación sobre derechos humanos está cubierto. Ahora bien, además, aparecen numerosos elementos vinculados con la educación a través de los derechos humanos y para los derechos humanos. De hecho, se aclara explícitamente que "el solo reconocimiento de los derechos es un importante avance, pero esta obligación no se termina con la existencia de un orden normativo, constituciones, leyes y tratados. Para hacer posible que los Derechos Humanos sean efectivos, se requiere una conducta gubernamental que asegure la existencia, en la realidad, de una eficaz garantía del libre y pleno ejercicio. Y para ello es imprescindible una ciudadanía comprometida que reclame su vigencia y contribuya a su efectividad" (PCI de Educación Primaria, 2015, 105). 
El PCI también indica en esta asignatura que la intención es abordar los contenidos “desde una perspectiva crítica y contextualizada, que permita analizar la vida social y política desde el marco de los derechos humanos, reconocer sus violaciones, las responsabilidades por su cumplimiento y las políticas públicas que los afirman y buscan garantizarlos" (PCI de Educación Primaria, 2015, 107). Más aún, se hace también explícita la intención de educar para la transformación y para "la construcción de un mundo justo, con dignidad y respeto" (PCI de Educación Primaria, 2015, 108), y se propone la utilización de recursos y estrategias para la enseñanza como la literatura, el cine, la televisión, sitios en Internet o visitas a lugares significativos de las luchas por la vigencia los derechos humanos.

Para finalizar, el PCI engloba el concepto de educación a través de y para los derechos humanos y explica la importancia de formar a los docentes adecuadamente para esta tarea tan fundamental y compleja: “debe concederse importancia primordial a la formación del docente en relación con la coherencia entre lo que se enseña, y las prácticas a través de las cuales se realiza esa enseñanza. Es necesario preparar a los docentes para que, en el ejercicio de la ciudadanía, la ética y los derechos humanos no se conviertan en un conjunto de declaraciones, sino en herramientas eficaces para la transformación social en búsqueda de un aula, una comunidad y un mundo con más paz, igualdad, equidad, justicia y respeto mutuo" (PCI de Educación Primaria, 2015, 107). En conclusión, esta asignatura aparece como el elemento más destacable del Campo de Formación Específica del PCI.

Por último, con respecto a las otras unidades curriculares, Tecnologías de la Información y la Comunicación en la Educación Primaria tiene en el DCJ algunos elementos de inclusión social, acceso a la tecnología y acceso a la información, pero no hace referencia específica a cuestiones de discriminación, bullying, etc. En el PCI, esta unidad tampoco está muy desarrollada en cuanto a temáticas de derechos humanos y desarrollo sostenible. En Sujetos de la Educación Primaria aparecen en el DCJ y en el PCI algunos contenidos sobre complejidad social y diversidad cultural, aunque no hay ninguna vinculación explícita a los derechos humanos. En Problemáticas de la Educación Primaria, se detectan propuestas de inclusión, integración, diversidad, igualdad de oportunidades y construcción democrática. 


\subsection{El Campo de la Formación en la Práctica Profesional}

Tabla 3: Campo de la Formación en la Práctica Profesional (DCJ Educación Primaria, 2014, 19)

TRAMOS

\begin{tabular}{c|l} 
Tramo 1: Sujetos y contextos de las prácticas & $\begin{array}{l}\text { Tramo 3: La residencia, momento de } \\
\text { intervención, reflexión e integración }\end{array}$
\end{tabular}

Tramo 2: Las intervenciones docentes en contextos reales

Espacio de Definición Institucional

El Campo de Formación en la Práctica Profesional es quizás el que menos contenidos puede ofrecer en materia de educación sobre derechos humanos, pero al mismo tiempo presenta buenas oportunidades para incluir la educación a través de los derechos humanos y también la educación para los derechos humanos. Sin embargo, en el DCJ, por más que aparecen algunos elementos aislados sobre diversidad, no hay referencias sobre estos puntos. El PCI tampoco incluye mayores elementos. Se habla de la flexibilidad, la reflexión y la crítica, y se recepta alguna mención sobre diversidad, pero nada explícito sobre educación en derechos humanos o en desarrollo sostenible. Justamente, acá se evidencia que todas las propuestas metodológicas, didácticas y pedagógicas que aparecen en los bloques de ciencias sociales y de ciencias naturales, y en la unidad curricular sobre ESI, no se reiteran en el CFPP. Al quedar descartadas en este campo y solo limitarse a uno o dos bloques del CFE o a una unidad del CFG, difícilmente se pueda conseguir de forma adecuada la pretendida transversalidad.

\subsection{Conclusiones sobre el currículo del Profesorado de Educación Primaria}

El Diseño Curricular Jurisdiccional del Profesorado de Educación Primaria no cuenta con ninguna materia específica sobre derechos humanos o sobre desarrollo sostenible. El Plan Curricular Institucional sí incluye en su programa la asignatura Ética, Derechos Humanos y Construcción de Ciudadanía. No obstante, más allá de este punto, a lo largo del DCJ y del PCI - sí aparecen contenidos relacionados con derechos humanos y desarrollo sostenible, aunque no siempre se mencionen así explícitamente ni se reiteren transversalmente.

En este sentido, predominan los elementos vinculados con la diversidad y la igualdad y no discriminación, pero también hay cuestiones de derechos laborales de los docentes, de democracia y ciudadanía, de adicciones (que no están receptados en el PCI) y se destaca particularmente la unidad sobre ESI. Como se advierte en la Tabla 4, hay más menciones 
sobre derechos humanos y desarrollo sostenible en el PCI que en el DCJ, y la mayoría de las referencias corresponden a los indicadores 2 (Democracia, ciudadanía, participación social y orden institucional) y 3 (Género, diversidad, inclusión y multiculturalismo). Luego, los indicadores con más menciones son sobre ambiente (indicador 5), sobre ESI y adicciones (indicador 7) y sobre derechos económicos, sociales, culturales y derechos colectivos (indicador 8). Prácticamente no hay referencias a la normativa sobre educación (indicador 1), a elementos de Memoria, Verdad y Justicia (indicador 6) y a derechos específicos de niñas, niños y adolescentes que no están incluidos dentro de los indicadores 3 o 7 (indicador 4). Con respecto al indicador 9, sobre derechos de las personas con discapacidad, si bien existen varias menciones hacia este grupo, se proponen más desde un foco de diversidad, igualdad e inclusión, que desde derechos propios del colectivo; por eso la mayoría de las referencias fueron anotadas dentro del indicador 3. Por último, el DCJ casi no menciona elementos de pedagogía de los derechos humanos (indicador 10) mientras que el PCI lo hace, aunque exclusivamente en el bloque de enseñanza de las ciencias sociales.

En cualquier caso, en pos de conseguir la enunciada transversalidad de los contenidos y valores de derechos humanos, debería estar más presente el enfoque o perspectiva de derechos a lo largo de todo el documento y no solo en los apartados particulares de ESI, Ética y Derechos Humanos y los bloques de Ciencias Naturales y Ciencias Sociales; aunque sean muy valiosas las referencias en estos espacios, si no se repiten en las distintas unidades, se corre el riesgo de que resulte insuficiente. Por ejemplo, tanto en el DCJ como en el PCI, los bloques de matemática y lengua, así como el CFPP, necesitarían abordar de forma más explícita la educación en derechos humanos. En este sentido, que en una materia tan relevante y troncal como matemática no aparezca nada sobre estas cuestiones pone en duda la efectividad de la tan pretendida transversalidad. Si quienes forman a los futuros docentes no les brindan herramientas o recursos pedagógicos para conectar su campo de enseñanza con los derechos humanos y el desarrollo sostenible, difícilmente lo puedan hacer luego los futuros maestros y profesores en sus clases. La educación en desarrollo sostenible en general tampoco ocupa un lugar relevante en el DCJ ni en el PCI, aunque existan algunas referencias en el indicador 5.

En definitiva, se podría concluir que tanto los institutos como los docentes de este profesorado cuentan con elementos en el DCJ y en el PCI donde anclarse si quisieran 
brindar contenidos de derechos humanos y desarrollo sostenible en sus clases. Sin embargo, esto no parece ser suficiente para garantizar el derecho a recibir esta clase de educación. No alcanza con que el Estado mencione en algunos apartados del DCJ la inclusión de ciertos contenidos temáticos relacionados con los derechos humanos, por más de que estos puntos sean valiosos y necesarios. Para efectivamente lograr esta clase de educación se necesita una propuesta más proactiva, un impulso más explícito, que ponga el tema en agenda y que no dependa de que los profesorados en su ámbito privado elijan brindar este tipo de contenidos. Por más que se reitere la pretendida transversalidad, ella no se ve reflejada en todas las distintas materias. Por último, se vuelve a aclarar que estas son conclusiones preliminares, que se limitan al estudio del currículo, y que forman parte de una investigación más amplia y profunda, que se completará con entrevistas y cuestionarios a docentes para ver si los contenidos que aparecen en el DCJ y en el PCI se trasladan a las clases de los profesorados, si se agregan otros, y si luego quienes adquieren estas herramientas las usan en sus clases con niños y niñas de primaria.

Tabla 4: Elementos e Indicadores en los PCJ y los DCI.

\begin{tabular}{|c|c|c|c|c|}
\hline CARRERA & \multicolumn{2}{|r|}{ DCJ } & \multicolumn{2}{|r|}{ PCI } \\
\hline & $\begin{array}{c}\text { ¿Contiene } \\
\text { elementos } \\
\text { de } \\
\text { Derechos } \\
\text { Humanos } \\
\text { / } \\
\text { Desarrollo } \\
\text { Sostenible } \\
?\end{array}$ & $\begin{array}{l}\text { Indicadores } \\
\text { Temáticos }\end{array}$ & $\begin{array}{c}\text { ¿Contiene } \\
\text { elementos de } \\
\text { Derechos } \\
\text { Humanos / } \\
\text { Desarrollo } \\
\text { Sostenible? }\end{array}$ & Indicadores Temáticos \\
\hline $\begin{array}{c}\text { Profesorad } \\
\text { o de } \\
\text { Educación } \\
\text { Primaria }\end{array}$ & SÍ & 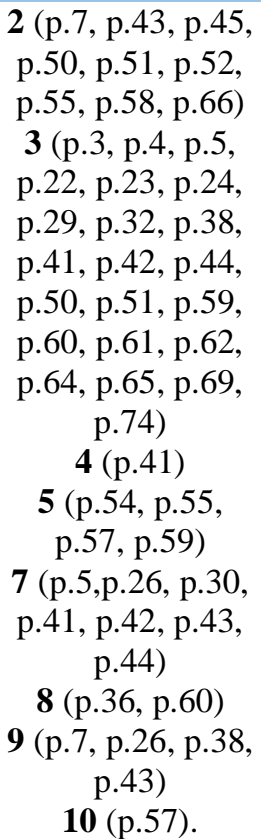 & SÍ & $\begin{array}{c}1 \text { (p.104) } \\
2 \text { (p.12, p.34, p.38, p.44, p.46, p.61, } \\
\text { p.65, p.67, p.89, p.91, p.99, p.101, } \\
\text { p.104, p.105, p.106, p.107, p.108, } \\
\text { p.109, p.110, p.113) } \\
\text { 3 (p.18, p.19, p.20, p.21, p.27, p.28, } \\
\text { p.41, p.43, p.45, p.64, p.91, p.94, p.98, } \\
\text { p.99, p.101, p.104, p.106, p.108, p.109, } \\
\text { p.111, p.114, p.115, p.116, p.126) } \\
\text { 4. (p.42, p.109, p.110) } \\
\text { 5 (p.75, p.76, p.82, p.84, p.93, p.104, } \\
\text { p.106, p.107, p.110) } \\
\text { 6. (p.26, p.109) } \\
\text { 7. (p.5, p.11, p.42, p.43, p.44, p.45, } \\
\text { p.46) } \\
\text { 8. (p.26, p.38, p.39, p.40, p.51, p.94, } \\
\text { p.104, p.107, p.110) } \\
\text { 9(p.3) } \\
\text { 10. (p.36, p.89, p. } 90, \text { p.91, p.95, p.98, } \\
\text { p.102, p.103, p.105, p.107, p.109). }\end{array}$ \\
\hline
\end{tabular}




\section{Palabras finales}

La educación en derechos humanos y desarrollo sostenible es un derecho. Pero también es una herramienta para garantizar la dignidad de las personas, que busca mejorar la convivencia y el progreso humano, teniendo en cuenta el cambio climático, la reducción de la pobreza, el consumo sostenible, la paz y la seguridad (ACEVEDO y BÁEZ, 2018). Además, esta formación contribuye a desarrollar una mayor conciencia participativa, una mejor comprensión de la sociedad, un aumento de la sensibilización con respecto a la naturaleza y una mayor responsabilidad en relación con el mundo que los rodea (UNESCO, 2016b).

Sin embargo, a pesar de que los Estados están obligados a garantizar la educación en derechos humanos y en desarrollo sostenible, y de que existe un gran consenso sobre los beneficios de fomentar esta enseñanza, la mayoría de los países no cumple apropiadamente con este mandato. Por ejemplo, solamente la mitad de los Estados miembros de UNESCO reportaron haber integrado la educación en desarrollo sostenible en políticas públicas relevantes (UNESCO, 2016c). En palabras recientes de esta institución, "el mundo aún está lejos de poder cumplir los compromisos internacionales de educación" (UNESCO, 2019, 12).

En consecuencia, el análisis de la formación docente en estos temas se vuelve fundamental. Desde ya, al estudiar la formación de maestros de primaria, un momento propicio para impulsar estos conocimientos, el estudio del DCJ no es suficiente ni definitorio. Los propios Lineamientos Nacionales advierten sobre esta limitación al reconocer que los diseños curriculares no alcanzan por sí solos para sostener procesos y modificar resultados de la educación (Resolución CFE N²4/07, 2007). Ahora bien, si ya desde el DCJ hay falencias, no es sencillo corregirlas a través de otros mecanismos.

En cualquier caso, se vuelve a destacar que el DCJ del profesorado de educación primaria de la Ciudad de Buenos Aires sí contiene elementos vinculados con la educación en derechos humanos, aunque no presente una materia específica al respecto (como sí lo hace el PCI) ni incluya en la mayoría de las materias un enfoque de derechos que favorezca su incorporación transversal. Las próximas fases de la investigación buscarán analizar la incidencia de los contenidos de derechos humanos y desarrollo sostenible que 
están presentes en el DCJ y en el PCI, es decir, si efectivamente se trasladan a las clases, si los futuros docentes cuentan con herramientas para enseñar esta clase de temas que pueden ser sensibles y si se respeta el enfoque de derechos.

Para finalizar, también se hace necesario reconocer que a pesar de la nobleza y ambición de sus objetivos, la educación en derechos humanos y desarrollo sostenible no podrá mejorar el mundo por sí sola, ni desde el ámbito de la educación ni desde el ámbito del derecho. Además de tratarse de una tarea sumamente compleja y exigente, para hacer realidad estas intenciones es fundamental un cambio estructural y cultural, que si bien puede estar influido por todos los niveles del sistema educativo formal y de las normas, también se construye según los medios de comunicación, las familias, religiones, tradiciones, amistades, etcétera (educación no formal e informal). Entonces, la educación formal podrá ser una herramienta importante y significativa para generar un cambio cultural y para mejorar el mundo, pero no necesariamente será siempre la principal, la única, la mejor o la más efectiva, al igual que puede suceder con el derecho, la política, la economía, la sociología o hasta los medios de comunicación y las redes sociales. El punto es que el potencial que puede tener la educación en derechos humanos y desarrollo sostenible y las leyes que la regulan no debe generar expectativas desmedidas, y será necesario trabajar en conjunto con otras disciplinas y otros ámbitos para alcanzar sus objetivos.

\section{Referencias bibliográficas}

ACEVEDO SUÁREZ, A. y BÁEZ PIMIENTO, A. (2018). La educación en cultura de paz. Herramienta de construcción de paz en el posconflicto. Revista Reflexión Política, 20(40), 68-80. Universidad Autónoma de Bucaramanga, Colombia. DOI: https://doi.org/10.29375/01240781.3455.

ALLIAUD, A. y VEZUB, L. (2012). El oficio de enseñar: sobre el quehacer, el saber y el sentir de los docentes argentinos. Revista Diálogo Educacional, 12(37). Pontifícia Universidade Católica do Paraná, Brasil. DOI: 10.7213/dialogo.educ.7211

Asamblea General de las Naciones Unidas (2018). Informe de la Relatora Especial sobre el derecho a la educación, A/73/262. Ginebra: ONU. 
Asamblea General de las Naciones Unidas (2020). Informe del Relator Especial sobre la cuestión de las obligaciones de derechos humanos relacionadas con el disfrute de un medio ambiente sin riesgos, limpio, saludable y sostenible, A/75/161. Ginebra: ONU.

BACHMANN, L. (2008). La Educación Ambiental en Argentina, hoy. Documento Marco sobre Educación Ambiental elaborado por la Dirección Nacional de Gestión Curricular y Formación Docente Áreas Curriculares del Ministerio de Educación de la República Argentina. Disponible en http://www.bnm.me.gov.ar/giga1/documentos/EL001599.pdf.

BARAJAS, L. (2012). Estudio de caso: una estrategia para la enseñanza de la Educación ambiental, Revista Praxis \& Saber, 3(5), 53 - 78. Universidad Pedagógica y Tecnológica de Colombia. DOI: https://doi.org/10.19053/22160159.1133.

CARMONA LARA, M. C. (2010). Derechos Humanos y Medio ambiente. En J. U. Carmona Tinoco, J. y Hori Fojaco, J. (Coords.), Derechos Humanos y Medio Ambiente (1 - 34). México: Universidad Nacional Autónoma de México.

Comisión Interamericana de Derechos Humanos (CIDH). (2019). Informe Empresas y Derechos Humanos: Estándares Interamericanos. Washington DC, Estados Unidos.

Comité de Derechos Económicos, Sociales y Culturales (1999). Observaciones generales 13: El derecho a la educación, E/C.12/1999/10. Ginebra: ONU.

Comité para la Eliminación de la Discriminación contra la Mujer (2017). Recomendación general 36 sobre el derecho de las niñas y las mujeres a la educación, CEDAW/C/GC/36. Ginebra: ONU.

CONDENANZA, L. y CORDERO, S. (2013). Educación Ambiental y legislación educativa en Argentina. Hacia un estado de la cuestión. Praxis Educativa, XVII (1), 
47-55. Facultad de Ciencias Humanas de la Universidad Nacional de La Pampa, Argentina.

Consejo Federal de Educación (2007). Lineamientos Curriculares Nacionales para la Formación Docente Inicial. Resolución CFE $\mathrm{N}^{\circ}$ 24/07. Disponible en http://www.bnm.me.gov.ar/giga1/normas/RCFE_24-07.pdf.

Corte Interamericana de Derechos Humanos (Corte IDH). (2017). Medio ambiente y derechos humanos. Opinión Consultiva 23/17. San José de Costa Rica.

Corte Interamericana de Derechos Humanos (Corte IDH). (2020). Caso Comunidades Indígenas Miembros de la Asociación Lhaka Honhat (Nuestra Tierra) Vs. Argentina. Sentencia de Fondo, Reparaciones y Costas. San José de Costa Rica.

GONZÁLEZ GAUDIANO, E. (2005). Educación Ambiental para el desarrollo Sustentable en América Latina y el Caribe. En Priotto, G. (comp.). Educación Ambiental para el Desarrollo Sustentable. Buenos Aires, Miño y Dávila Editores.

GIL CANTERO, F. (1991). La enseñanza de los derechos humanos, Revista española de pedagogía, 49 (190). Universidad Internacional de La Rioja, España.

KLAINER, R. y FERNÁNDEZ, M. (2008). La educación en derechos humanos en la Argentina: ideas-fuerza de los años ochenta a la actualidad. En Magendzo, A. (ed.). Pensamiento e ideas-fuerza de la educación en derechos humanos en Iberoamérica. Santiago de Chile, Editorial SM.

Instituto Danés de Derechos Humanos. (2013). Recursos para la educación en Derechos Humanos. Dinamarca. en https://www.humanrights.dk/files/media/dokumenter/udgivelser/hre_esp.pdf.

Instituto Interamericano de Derechos Humanos (2004). III Informe Interamericano de la Educación en Derechos Humanos: Desarrollo en la formación de educadores. San José de Costa Rica. 
Instituto Interamericano de Derechos Humanos (IIDH). (2013). El Derecho a la Educación en Derechos Humanos en las Américas. San José de Costa Rica.

Institutos Públicos de Profesorado de Educación Primaria de la Ciudad Autónoma de Buenos Aires (2015). Plan Curricular Institucional para la Formación Docente del Profesorado de Educación Primaria. Disponible en https://www.buenosaires.gob.ar/sites/gcaba/files/pci_prof_educ_primaria.pdf.

MAGENDZO, A. y TOLEDO-JOFRÉ, M. (2015). Educación en derechos humanos: Estrategia pedagógica-didáctica centrada en la controversia. Revista Electrónica Educare, 19(3), 1-16.

MASTACHE, A. (2019). La formación ciudadana como contenido transversal en la escuela secundaria. Aportes a su didáctica. Revista del IICE (46), 171-186. DOI: https://doi.org/10.34096/iice.n46.8597.

MEDICI, A. (2018). Articulación de docencia-investigación-extensión para una práctica pedagógica crítica en la enseñanza de los Derechos Humanos. Revista Derechos en Acción, 7(7), 126-145.

Ministerio de Educación de la Ciudad Autónoma de Buenos Aires (2014). Diseño Curricular Jurisdiccional para la Formación Docente del Profesorado de Educación Primaria. Resolución-2514-2014-MEGC. Disponible en https://www.buenosaires.gob.ar/sites/gcaba/files/res_2514_fd_edu_primaria_4.pdf.

Oficina del Alto Comisionado de las Naciones Unidas para los Derechos Humanos (ACNUDH) y United Nations Educational, Scientific and Cultural Organization (UNESCO) (2012). Programa Mundial para la Educación en Derechos Humanos. Segunda Etapa: Plan de Acción, HR/PUB/12/3. Nueva York y Ginebra: ONU.

REQUESENS GALNARES, A. (2006). Retos a la educación en derechos humanos. En Educación en Derechos Humanos, Programa de Cooperación sobre Derechos Humanos México-Comisión Europea. en http://ru.juridicas.unam.mx/xmlui/handle/123456789/28222. 
RONCONI, L. (2017). La enseñanza en derechos humanos en las Facultades de Derecho en Argentina: desafíos pendientes. Revista Pedagogía Universitaria y Didáctica del Derecho, 4(1), 5-37.

TIBBITTS, F. (2017). Evolution of Human Rights Education Models. En Bajaj, M. (Ed.), Human Rights Education: Theory, Research, Praxis, Estados Unidos: University of Pennsylvania Press.

Tribunal Europeo de Derechos Humanos (TEDH). (2005). Caso Leyla Şahin v. Turquía. Estrasburgo, Francia.

Tribunal Europeo de Derechos Humanos (TEDH). (2014). Caso Velyo Velev vs. Bulgaria. Estrasburgo, Francia.

Tribunal Europeo de Derechos Humanos (TEDH). (2016). Caso Çam v. Turquía. Estrasburgo, Francia.

United Nations Educational, Scientific and Cultural Organization (UNESCO) (2016a). Action for Climate Empowerment: Guidelines for accelerating solutions through education, training and public awareness. Francia: UNESCO.

United Nations Educational, Scientific and Cultural Organization (UNESCO) (2016b). Formando el futuro que queremos. Decenio de las Naciones Unidas de la Educación para el Desarrollo Sostenible (2005-2014): Informe Final. Francia: UNESCO.

United Nations Educational, Scientific and Cultural Organization (UNESCO). (2016c). Education 2030: Incheon Declaration and Framework for action for the implementation of Sustainable Development Goal 4. Ensure inclusive and equitable quality education and promote lifelong learning opportunities for all. Disponible en https://unesdoc.unesco.org/ark:/48223/pf0000245656. 
United Nations Educational, Scientific and Cultural Organization (UNESCO) (2019). Cumplir los compromisos: ¿van los países por el buen camino para alcanzar el ODS 4? Disponible en https://unesdoc.unesco.org/ark:/48223/pf0000369009_spa.

VEZUB, L. (2011). ¿Qué cuentan las trayectorias de desarrollo profesional de los docentes sobre su oficio? En A. Alliaud y D. Suárez (coords.). El saber de la experiencia. Narrativa, investigación y formación docente. Buenos Aires: FFYL, UBA-CLACSO.

VEZUB, L., y GARABITO, F. (2017). Los profesores frente a la ¿nueva / vieja? Escuela secundaria argentina. Revista Electrónica de Investigación Educativa, 19 (1), 123-140. Universidad Autónoma de Baja California, Ensenada, México. DOI: https://doi.org/10.24320/redie.2017.19.1.1096 